\title{
Anesthesia- patient's apprehension, concerns, and counseling
}

\author{
Mishra C. ${ }^{1 *}$, Saxena . $^{2}$, Dubey A. ${ }^{3}$ \\ DOI: https://doi.org/10.17511/ijmrr.2020.i04.03 \\ 1* Chandra Shekhar Mishra, Assistant Professor, Department of Anaesthesia, LNMC and JK Hospital, Bhopal, Madhya Pradesh, India. \\ 2 Sushant Saxena, Final Year Postgraduate, Department of Anaesthesia, LNMC and JK Hospital, Bhopal, Madhya Pradesh, India. \\ 3 Anuj Dubey, Assistant Professor, Department of Anaesthesia, LNMC and JK Hospital, Bhopal, Madhya Pradesh, India.
}

Introduction: To allay anxiety and resolve the patient's apprehension regarding general anesthesia through counseling. Patients presenting to PAC were asked to participate in a 2-way conversation meant to evaluate and study his apprehension, concerns in relation to anesthesia, and subsequently, a counseling session was given to answer his doubts and resolve his fear. Material and Methods: In the study, it was found the fear of death was the greatest concern. The other major concerns were fear of paralysis and heart attack. Results: The level of fear decreased as the severity of surgery decreased. For major surgery, the fear of death differed significantly with age, especially with increasing age came a lessened fear of death. Counseling by anesthesia provider regarding the fear that the patient reported help to reduce the anxiety. It allows for a more personalized approach towards the patient. It helps anesthesia provider to tailor the informed consent process. Conclusion: In general, the study increases awareness regarding anesthesia among patient and their relatives.

Keywords: General Anaesthesia, Fear of anesthesia, Patient's counseling

\section{Corresponding Author}

Chandra Shekhar Mishra, Assistant Professor, Department of Anaesthesia, LNMC and JK Hospital, Bhopal, Madhya Pradesh, India.

Email: dr.chandrashekharm@gmail.com
How to Cite this Article

Chandra Shekhar Mishra, Sushant Saxena, Anuj Dubey, Anesthesia- patient's apprehension, concerns, and counseling. Int J Med Res Rev. 2020;8(4):302-306.

Available From

https://ijmrr.medresearch.in/index.php/ijmrr/article/ view/1204
To Browse

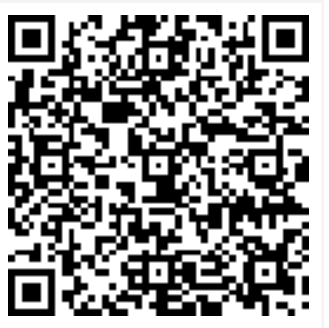

\section{Introduction}

Anesthesia is commonly recognized as a practice that makes the patient unaware of their environment during a surgical procedure. Anesthesia aims to relieve the patient's unbearable pain during the surgery that may potentiate extreme physiologic disturbances and result in harsh memories. The thought of undergoing general anesthesia strikes terror in the hearts of many people. During the
Manuscript Received 2020-07-29

Conflict of Interest No

Review Round 1
2020-08-12
Funding
Nil
$\mathrm{Nil}$
Preoperative

assessment,

regular communication is required with the patients whose main fear is neither of the surgery, nor of the prospect of pain after the surgery or of the prospect of complications, or death after the surgery, but an immense fear of general anesthesia [1]. This fear of general anesthesia is not rare. Studies by psychiatrists reveal that about $30 \%$ of people are more fearful of general anesthesia than the surgery itself. People have always been scared of general () 2020 by Chandra Shekhar Mishra, Sushant Saxena, Anuj Dubeyand Published by Siddharth Health Research and Social
Welfare Society. This is an Open Access article licensed under a Creative Commons Attribution 4.0 International License https://creativecommons.org/licenses/by/4.0/ unported [CC BY 4.0].

Accepted 2020-08-25 
Anesthesia. Many voice fears they will not wake up from this "induced sleep? ?. Some anxious patients might even elect to cancel their surgery just because of the qualms that they have of undergoing general anesthesia [1]. It is normal for one to be nervous when thinking that they cannot be in control of their own body.

Previous studies have shown that the majority of the patients feel anxious toward anesthesia. The main sources of anxiety and fear in patients before the operation stems from the concern of the anesthesia itself rather than the surgical procedure [2], pain during or after the procedure [3]. Administering pre-anesthetic anxiolytic medication is a popular and effective method used to reduce the apprehension of patients who are otherwise prepared to undergo surgery. Anxiolytic medications such as midazolam, are commonly used as a preanesthetic relief in stressed and apprehensive patients $[4,5]$.

The purpose of this study is to identify the concerns of patients regarding general anesthesia prior to their surgeries, assess the main sources of patient's distress prior to anesthesia (including postoperative pain, fear of death, nausea, needle phobia, paralysis, awareness during surgery, and the worry over the chance of disclosure of personal information).

\section{Materials and Methods}

Setting: Laxmi Narayan Medical College and JK Hospital, Bhopal (Central India)

Duration and Type of Study: Observational crosssectional survey conducted between January 2020 to June 2020

Sampling method: Patients were selected randomly without any bias for age, sex, religion, and type of surgery.

Sample size calculation: This study included 200 patients scheduled for surgery in the pre-anesthesia clinic (PAC). All the patients coming in the PAC must be included based on inclusion criteria.

Inclusion criteria: Adult patients of either gender between 18 to 60 years of age, Patients belonging to ASA physical status 1 or 2, and Patients scheduled for routine elective surgery requiring general anesthesia.

Exclusion criteria: Refusal of consent, Patient Age $<18$ or $>60$ years, and Patients belonging to ASA
Physical status 3 or 4 .

Data collection procedure: All the patients coming to the PAC were asked to participate in a two-way conversation which was meant to evaluate the apprehension and concerns they had for general anesthesia. These patients were asked if they have any doubts or queries regarding the wider issues of concerns and anxiety associated with the hospital and operating room environment, hospital personnel, and general anesthesia. However, this study only considered the queries relating to patient anxiety when experiencing general anesthesia.

Study procedure: They were subsequently counseled by the anesthesiologist regarding the procedure they will be undergoing. The anesthesiologist talked to the patients and informed them about the anesthetic procedures during general anesthesia. All the queries of the patients were answered so as to remove any doubt or fear from the patient's mind prior to the surgery.

Ethical consideration and permission: Written informed consent was obtained from all participants and this study was approved by the institutional ethical committee.

\section{Results}

Table-1: Concerns that made patients anxious.

\begin{tabular}{|l|l|}
\hline a) & Dying while asleep \\
\hline b) & The thought of not waking up \\
\hline c) & Waking up during surgery \\
\hline d) & The experience of being awake \\
\hline e) & Possibly feeling the surgeon's touch \\
\hline f) & $\begin{array}{l}\text { Seeing their body cut open or surgery is more painful than expected } \\
\text { were anxiety-provoking aspects }\end{array}$ \\
\hline g) & Anxiety while waiting to go into surgery or arriving at the theatre \\
door
\end{tabular}

27 percent of respondents said they were very or extremely anxious, 29 percent said they were quite anxious, 36 percent said they were a little anxious and 08 percent experienced no anxiety at all.

Patients were asked to indicate their anxiety levels about different issues. This showed that:

- The top three concerns that made patients very anxious were the thought of not waking up (21per cent), dying while asleep (35 percent) and waking up during surgery (16 percent). 
- When combined, all the patients who were anxious, the top five concerns were: waiting for their turn in theatre (63 percent), the thought of arriving at the theatre door (51 percent), dying while asleep or not waking up afterward (both 68 percent) and waking up during surgery (49 percent).

- Forty-five percent said that they didn't like the thought of having to put their trust in strangers and 15 percent felt very anxious about this.

- Anxiety levels were lowest when it came to interactions with medical staff and the support of a partner or friend. Thirty-five percent felt very calm about the anesthetist explaining the procedure, 23 percent about the anesthetist visiting, and 19 percent about the nurse explaining the procedure. Twenty-one percent felt very calm about having a friend or partner with them during recovery.

Though, while patients need less physical nursing care, our survey shows that more attention needs to be paid to the psychological aspects of their care.

The formal and timely provision of information about the planned surgery- together with a patientcentered approach to the provision of information, such as pre-assessment clinics- is a vital first step.

It is very clear from our survey that numerous patients do not know how the anesthesia procedure works and that this has led to misapprehensions about, for example, waking up during surgery. It is very important to tackle these fallacies if patient anxiety is reduced before the day of elective surgery."

\section{Discussion}

A person undergoing general anesthesia is anything but nearly dead, or in a death-like state. General anesthesia is essentially very safe, and some desperately sick patients are in the improved condition under general anesthesia than when they are awake and breathing by themselves. Their vital functions, such as blood pressure, the amount of blood pumped by their hearts, etc. are improved by the anesthesia, extra medicines, and extra oxygen administered by the anesthesiologist.

People under general anesthesia are different from being nearly dead. Instead, they are very much alive, though they are unconscious, unable to speak, unable to move, unable to breathe, and unable to respond in any way to external stimuli.
So, it can be safely assumed that general anesthesia is not equal to near death.

Safety of General Anaesthesia: So what are the probabilities of dying as a consequence of general anesthesia and other events. Look at the probability of dying as a result of general anesthesia, pregnancy, and some common planned operations.

Chance of dying as a result of pregnancy $=10-15$ deaths per 100,000 live births $(0.01-0.015 \%)$ [6].

Chance of dying as a result of general anesthesia alone $=$ somewhat less than $11-16$ deaths per 100,000 persons $(0.01-0.016 \%)[7,8]$.

Chance of dying due to surgical complications of removing the gallbladder $=150$ to 1400 deaths per 100,000 operations, depending upon the health of the patient and technique of surgery $(0.15-1.4 \%)$ $[9,10]$.

Chance of dying due to complications resultant of the operation of surgically removing the large bowel due to cancer $=800$ to 5000 deaths per 100,000 operations, depending upon the health and technique of surgery $(0.8-5.0 \%)$ [11].

This is an illustration of how public opinion, sociocultural factors, and rumors determine perceptions.

Women were blissfully happy during their reproductive lives when they learned that they are pregnant, a state whose mortality is very much comparable to that of general anesthesia. They were ecstatic with pregnancy but scared of anesthesia.

Curious, I had explained these things, hoping to comfort these women, while explaining at the same time that statistics and emotions are two different things. The same principles apply to the gallbladder and large bowel operations undergone by women.

As regards the men who undergo gallbladder and large bowel operations, they were often also ecstatically happy when their sisters, wives, or daughters announced a pregnancy. In other words, they were happy with the risks undergone by these women but unhappy with anesthesia which has similar mortality. Also a strange attitude.

Even so, I never told these people about the mortality of the other types of surgery they are about to undergo - this is the responsibility of the surgeon.

"Our survey underlines the importance of patients 
Receiving planned and timely information about anesthesia, prior to the day of surgery, in order to limit their anxiety," says Dr. Mark Mitchell, senior lecturer in the Faculty of Health and Social Care at the University of Salford, UK $[12,13,14,15]$.

"Undergoing day surgery and general anesthesia is very common," says Dr. Mitchell. "The development of less invasive techniques means that the surgical effects on the body are now markedly reduced and, as a direct consequence, the amount of physical nursing care required before and after surgery is also considerably reduced $[12,13,14,15]$.

Wallace (1984) advocated the use of psychological preparation to allay the anxiety preoperatively [16].

Betty J. Blythe, Jolene C. Erdahl (1986) highlighted the use of stress inoculation to counter a patient's anxiety and depression before open-heart surgery [17].

Devine (1992) encouraged the effects of psychoeducational care on the recovery, postsurgical pain, and psychological distress of adult surgical patients [18].

For medically fit patients undergoing a planned surgery, general anesthesia is about as dangerous as pregnancy in a medically fit woman. In other words, general anesthesia is very safe.

However, the poorer the health of a person, the older they are, and the higher the risk of the operation - the greater the chance of dying as a result of anesthesia and surgery.

There are actually very few conditions where anesthesia is likely to be lethal for a patient, e.g. extremely severe aortic stenosis, major coronary artery stenosis, someone in deep shock, etc.

Fortunately, these conditions occur very seldom. In general, for nearly all patients, as the aforementioned discussion undoubtedly demonstrates, anesthesia is very safe and far less hazardous than the effects of surgery.

\section{Limitation}

The only limitation of the present study include a small sample size and of a particular region. If it occurs on a large scale (i.e. large number of participants of different state), then more queries about the anesthesia will be added, amongst the population.

\section{Conclusion}

The aim of the study was to reveal the aspects patients find anxiety aggravating while undergoing surgery under general anesthesia. One important area identified which gave rise to considerable anxiety for the majority of patients - provision of accurate preoperative anesthetic information. Therefore, it can be positively stated that anxiety prior to general anesthesia will be associated with a lack of accurate information (length of anesthesia, the period of unconsciousness) and misconceptions regarding intra-operative events (feeling, seeing, hearing surgery).

\section{What does this study add to the existing knowledge?}

It is suggested that communication should take place prior to the day of surgery to help discover and dissipate misapprehensions and underline safe, controlled anesthesia. In addition, the utilization and development of a 'patient-friendly' theatre environment must be pursued. Future studies may wish to investigate a comparison between the uses of a dedicated nurse, distracting techniques, patient-friendly theatre environments.

\section{Authors Contribution}

\author{
Dr. C.S. Mishra: Concept, Study design \\ Dr. Sushant Saxena: Data Analysis \\ Dr. Anuj Dubey: Manuscript preparation
}

\section{Reference}

01. Kain ZN, Wang SM, Mayes LC, Caramico LA, Hofstadter MB. Distress during the induction of anesthesia and postoperative behavioral outcomes. Anesth Analg. 1999;88(5)10421047.

[Crossref]

02. Mendl G. The effect of the preanesthetic interview on anxiety prior to minor interventions. Cah Anesthesiol. 1990;38(4)237239.

[Crossref]

03. Egan KJ, Ready LB, Nessly M, Greer BE. Selfadministration of midazolam for postoperative anxiety- A double blinded study. Pain. $1992 ; 49(1) 3-8$.

[Crossref] 
04. Mavridou $P$, Dimitriou $V$, Manataki A, Arnaoutoglou E, Papadopoulos G. Patient's anxiety and fear of anesthesia- Effect of gender, age, education, and previous experience of anesthesia- A survey of 400 patients. J Anesth. $2013 ; 27(1) 104-108$.

[Crossref]

05. Kogan A, Katz J, Efrat R, Eidelman LA. Premedication with midazolam in young children- A comparison of four routes of administration. Paediatr Anaesth. 2002;12(8)685-689.

[Crossref]

06. Berg CJ, Chang J, Elam-Evans L, Flowers L, Herndon J, Seed KA, Syverson CJ. Pregnancyrelated mortality surveillance-United States. MMWR Surveill Summ. 1991-1999.

[Crossref]

07. Lienhart A, Auroy $Y$, Péquignot $F$, Benhamou $D$, Warszawski J, Bovet $M$, et al. Preliminary results from the SFAR-iNSERM inquiry on anaesthesiarelated deaths in France- mortality rates have fallen ten-fold over the past two decades. Bulletin De L'academieNationale De Medecine. 2004;188(8)1429-1437.

[Crossref]

08. Arbous MS, Meursing $A E$, van Kleef JW, de Lange JJ, Spoormans $\mathrm{HH}$, Touw $\mathrm{P}$, et al. Impact of anesthesia management characteristics on severe morbidity and mortality. Anesthesiol- J Am Soc Anesthesiol. 2005;102(2)257-268. [Crossref]

09. Feldman MG, Russell JC, Lynch JT, Mattie A. Comparison of mortality rates for open and closed cholecystectomy in the elderlyConnecticut statewide survey. J Laparoendos Surg. 1994;4(3)165-172.

[Crossref]

10. Hannan EL, Imperato PJ, Nenner RP, Starr $H$. Laparoscopic and open cholecystectomy in New York State- mortality, complications, and choice of procedure. Surg. 1999;125(2)223-231.

[Crossref]
11. Mitchell MJ. Patients perceptions of preoperative preparation for day surgery. J Advan Nurs. 1997;26(2)356-363.

[Crossref]

12. Mitchell MJ. Anxiety management- A distinct nursing role in day surgery. Ambulat Surg. 2000;8(3)119-128.

[Crossref]

13. Mitchell MJ. Anxiety Management in Adult Day Surgery- A Nursing Perspective. University of Salford Manchester. 2005.

[Crossref]

14. Mitchell MJ. Conscious surgery- Influence of the environment on patient anxiety. J Advan Nurs. 2008;64(3)261-271.

[Crossref]

15. Wallace LM. Psychological preparation as a method of reducing the stress of surgery. J Human Stress. $1984 ; 10(2) 62-77$.

[Crossref]

16. Blythe BJ, Erdahl JC. Using stress inoculation to prepare a patient for open-heart surgery. Health and Social Work. 1986;11(4)265-274. [Crossref]

17. Devine EC. Effects of psychoeducational care for adult surgical patients- a meta-analysis of 191 studies. Patient education counselling. $1992 ; 19(2) 129-142$.

[Crossref]

18. Meyerhardt JA, Heseltine D, Niedzwiecki D, Hollis D, Saltz LB, Mayer RJ, Thomas J, Nelson $H$, Whittom R, Hantel A, Schilsky RL. Impact of physical activity on cancer recurrence and survival in patients with stage III colon cancerfindings from CALGB 89803. J Clin Oncol. 2006;24(22)3535-3541.

[Crossref] 